\title{
Grey scale thyroid ultrasonography in the evaluation of patients with Graves' disease
}

\author{
Paolo Vitti \\ Dipartimento di Endocrinologia, University of Pisa, Via Paradisa 2, 56124 Pisa, Italy
}

Graves' disease is a genetically induced autoimmune disorder characterized by hyperthyroidism due to circulating thyroid stimulating hormone (TSH) receptor autoantibodies (TRAb) with thyroid stimulating activity (1). Graves' disease is the most common cause of hyperthyroidism, which has an estimated prevalence in iodine sufficient areas of 20/1000 females and 2.3/ 1000 males (2). It is most common in females between the ages of 20 and 50 years, but it may occur at any age. The laboratory diagnosis of Graves' disease is based on the finding of high serum thyroid hormone and undetectable serum TSH concentrations associated with circulating thyroglobulin and thyroperoxidase antibodies. TRAb is detectable in almost $90 \%$ of patients, but usually it is not needed for the diagnosis. Radioisotope scan of the thyroid shows a diffuse and homogeneous radionuclide uptake.

Treatment strategies for Graves' hyperthyroidism include medical therapy with antithyroid drugs (ATD) or thyroid ablation with ${ }^{131}$ I or surgery. ATD, mainly thionamides, inhibit thyroid hormone synthesis by blocking iodine organification catalysed by thyroid peroxidase. Treatment of Graves' disease with thionamides is mainly performed in Europe and in Japan, while in the USA and Canada radioiodine is commonly preferred as the first choice treatment (3). Many studies have been undertaken to find parameters which help predict the clinical outcome of Graves' hyperthyroidism after ATD withdrawal. Among them, TRAb measurement at the end of an ATD course was found to be the most predictive $(4,5)$.

After its introduction into clinical practice in the late sixties, thyroid ultrasonography (US) proved to be very effective in the diagnostic approach to thyroid diseases, the anatomical location of the gland being advantageous for this technique. The most widely used application of thyroid US is identification and characterization of thyroid nodules (6). Rapid improvements in the development of US equipment have made available real-time high frequency transducers (7.5$10 \mathrm{MHz}$ ) with high resolution, which allow a more precise definition of the echostructure of the thyroid tissue. In addition, recently developed color Doppler technology allows determination of the blood flow through the gland, offering the possibility of recording objective measurements, such as the peak systolic velocity of the blood flow at the level of thyroid arterial vessels (i.e. inferior thyroid artery). Using these techniques, thyroid blood flow has been shown to be correlated with the thyroid status in patients with Graves' disease, being markedly increased in 17/18 patients with untreated active hyperthyroidism and less clearly increased in treated patients. In patients with Hashimoto's thyroiditis, the thyroid blood flow was not correlated to thyroid status or treatment and was never markedly increased (7). Color Doppler sonography was also shown to be useful in distinguishing cases of destructive thyrotoxicosis by amiodarone (i.e. amiodarone thyrotoxicosis type 2), characterized by a low thyroid blood flow, from cases in which thyroid hyperfunction also occurs (i.e. amiodarone thyrotoxicosis type 1), characterized by an increased thyroid blood flow (8).

With grey scale US, the normal thyroid has a peculiar echo density, making the gland well distinguishable from surrounding neck muscles. Thyroid echogenicity is due to the gland's follicular structure: the interface between thyroid cell and colloid exhibits high acoustic impedance, causing high frequency acoustic waves to be reflected back to the probe. Conditions that change the normal anatomical structure of the gland cause this echo pattern to be altered.

Espinasse (1983) and Gutekunst et al. (1989) were the first to report abnormal thyroid ultrasound patterns, characterized by a diffuse low echogenicity, in patients with Hashimoto's thyroiditis and Graves' disease (9, 10). Marcocci et al. (11) found a diffuse low echogenicity in the thyroids of $44 / 238$ patients with goiter and circulating thyroid autoantibodies. The degree of hypoechogenicity was significantly correlated with the levels of circulating thyroid autoantibodies. While thyroid function was normal in all 194 patients with normal thyroid echogenicity, hypothyroidism was found in $64 \%$ of those with thyroid hypoechogencity. Histology of excised thyroid tissue from patients who underwent surgery for tracheal decompression showed diffuse lymphocytic infiltration in patients with thyroid hypoechogenicity, while in patients with normal thyroid echogenicity a histological pattern of colloid goiter with focal thyroiditis was found. These data clearly showed that thyroid hypoechogenicity was due to diffuse thyroiditis and 
was correlated with hypothyroidism. In the same paper $59 / 90(65 \%)$ patients with Graves' disease were found to have a diffusely low thyroid echogenicity. While diffuse lymphocytic infiltration accounts for thyroid hypoechogenicity in Hashimoto's thyroiditis, in Graves' disease the hypoechogenic pattern may be due to reduced colloid content with increased cellularity and reduction of the cell-colloid interface and/or to the increased blood flow (12).

Using ultrasonography, Vitti et al. (13) reported that about $70 \%$ of patients with Graves' disease exhibit a low thyroid echogenicity. Whatever the reason for thyroid hypochogenicity in Graves' patients, this pattern is significantly associated with a higher frequency of TRAb positivity and with the relapse of hyperthyroidism. The study group included 105 patients who underwent a course of methimazole treatment. Thyroid ultrasonography was performed at diagnosis, and TRAb levels were measured at the end of treatment. During a 6-18 month follow-up period after methimazole treatment, $87 / 105(83 \%)$ patients had relapse of hyperthyroidism and 18/105 (17\%) were in remission. Recurrence of hyperthyroidism occurred in $71 / 76$ $(93 \%)$ patients with thyroid hypoechogenicity and in $16 / 29$ (55\%) of those with normal thyroid echogenicity. Positive TRAb values at the end of methimazole treatment were found in 59/76 (78\%) patients with thyroid hypoechogenicity and in $12 / 29(41 \%)$ patients with normal thyroid echogenicity. Sixty-five of eightyseven $(74 \%)$ patients with relapse of hyperthyroidism and $6 / 18(33 \%)$ of those who remained euthyroid were TRAb-positive at the end of methimazole treatment. The finding of thyroid hypoechogenicity at diagnosis had higher specificity (0.81) and sensitivity (0.72) compared with TRAb positivity at the end of methimazole treatment ( 0.74 and 0.66 , respectively) for the prediction of relapse of hyperthyroidism (13). Therefore, the evaluation of thyroid echographic pattern promised to be a useful prognostic tool in patients with Graves' disease.

Shieman et al. (14), studying 53 patients with Graves' disease, confirm that thyroid echogenicity is lower in these patients compared with that in 100 euthyroid volunteers. In agreement with the data of Vitti et al. (13), significantly lower echogenicity was found in patients with elevated TRAb levels and in those with active ophthalmopathy, suggesting that in some way this echographic pattern is associated with a more active disease. The merit of the paper of Shieman is the effort the authors made to obtain an objective measurement of thyroid echogenicity. To this purpose, the thyroid images obtained with a $7.5 \mathrm{MHz}$ real-time transducer were recorded, keeping the operating conditions constant, and in selected regions of the thyroid the grey scale density was evaluated and translated into a numerical scale. The intraassay and interassay variations of grey determination were $<5 \%$.
In the last few years, increasing evidence has been obtained indicating that thyroid US can be very useful in the diagnostic approach to thyroid autoimmune diseases, including Graves' disease. At present, thyroid US provides skilled operators with a useful method for diagnosing Graves' disease and for evaluating its severity. In the future, ultrasound imaging will prove increasingly useful as more sophisticated equipment is developed and operators increase their knowledge of color Doppler and three-dimensional technologies. Thus, ultrasound imaging is likely to increase in importance for diagnosis and follow-up of Graves' disease and become an essential technique for all thyroidologists.

\section{References}

1 Zakarija M \& McKenzie JM. The spectrum and significance of autoantibodies reacting with the thyrotropin receptor. Endocrinology and Metabolism Clinics of North America 198716 343-363.

2 Vanderpump MP, Tunbridge WM, French JM, Appleton D, Bates D, Clark F et al. The incidence of thyroid disorders in the community: a twenty-year follow-up of the Whickham Survey. Clinical Endocrinology 199543 55-68.

3 Reinwein D, Benker G, Lazarus JH, Alexander WD and the European multicenter study group on antithyroid drug treatment. A prospective randomized trial of antithyroid drug dose in Graves' disease. Journal of Clinical Endocrinology and Metabolism 199376 1516-1521.

4 Schleusener H, Schwander J, Fischer C, Holle R, Holl G, Badenhoop $\mathrm{K}$ et al. Prospective multicentre study on the prediction of relapse after antithyroid drug treatment in patients with Graves' desease. Acta Endocrinologica 1989120 689-701.

5 Vitti P, Rago T, Chiovato L, Pallini S, Santini F, Fiore E et al. Clinical features of patients with Graves' disease undergoing remission after antithyroid drug treatment. Thyroid 19977 369-375.

6 Hegedus L \& Karstrup S. Ultrasonography in the evaluation of cold thyroid nodules. European Journal of Endocrinology 1998138 30-31.

7 Vitti P, Rago T, Mazzeo S, Brogioni S, Lampis M, De Liperi A et al. 1995 Thyroid blood flow evaluation by color-flow doppler sonography distinguishes Graves' disease from Hashimoto's thyroiditis. Journal of Endocrinological Investigation 199518 857-861.

8 Bogazzi F, Bartalena L, Brogioni S, Mazzeo S, Vitti P, Burelli A et al. Color flow doppler sonography rapidly differentiates type I and type II amiodarone-induced thyrotoxicosis. Thyroid 19977 541-545

9 Espinasse P. L'echographie thyroidienne dans les thyroidites lymphocytaires chroniques autoimmunes. Journal of Radiology $198364537-544$.

10 Gutenkust R, Hafermann W, Mansky T \& Scriba PC. Ultrasonography related to clinical and laboratory findings in lymphocitic thyroiditis. Acta Endocrinologica 1989121 129135.

11 Marcocci C, Vitti P, Cetani F, Catalano F, Concetti R \& Pinchera A. Thyroid ultrasonography helps to identify patients with diffuse lymphocytic thyroiditis who are prone to develop hypothyroidism. Journal of Clinical Endocrinology and Metabolism 199172 209-213

12 Muller H W, Schroder S, Schneider C \& Seiffert G. Sonographic tissue characterisation in thyroid gland diagnosis. Klinische Wochenschrift 198663 706-710 
13 Vitti P, Rago T, Mancusi F, Pallini S, Tonacchera M, Santini F et al. Thyroid hypoechogenic pattern at ultrasonography as a tool for predicting recurrence of hyperthyroidism after medical treatment in patients with Graves' disease. Acta Endocrinologica $1992126128-131$.

14 Schiemann U, Gellner R, Riemann B, Schierbaum G, Menzel J, Domschke W et al. Standardized grey scale ultrasonography in
Graves' disease: correlation to autoimmune activity. European Journal of Endocrinology 1999141 332-336.

Received 30 August 1999

Accepted 9 September 1999 